\title{
Pigmented Bowen's disease associated with high-risk HPV simulating melanoma of the hand
}

\author{
Rute Facchini Lellis ${ }^{1}$ \\ Renata Diniz Jacques Gonçalves ${ }^{3}$
}

John Verrinder Veasey ${ }^{2}$

DOI: http:/ / dx.doi.org/10.1590/abd1806-4841.20176331

\begin{abstract}
Bowen's disease is an in situ squamous cell carcinoma of the skin with only $2 \%$ of pigmented cases reported. It is clinically characterized by papules and plaques of blackened surface that may be caused either by sun damage - usually in photoexposed areas in elderly individuals - or by human papillomavirus infection - usually in the anogenital region of young adults. Dermoscopic aspects of Bowen's disease are discussed for over a decade, but with no definitive criteria that would lead to a definitive diagnosis. We present a case of Bowen's disease affecting the finger of a 57-year-old Asian patient. The lesion clinically and dermoscopically simulated a melanoma. Histopathological findings suggested the diagnosis of pigmented Bowen's disease. Pigmented Bowen's disease should be considered a differential diagnosis of melanoma, since its clinical and dermoscopic criteria are unspecific. Histopathological examination remains the gold standard for the diagnosis of the disease. Keywords: Bowen's disease; Carcinoma in situ; Dermoscopy; Histology; Human papillomavirus 16; Human papillomavirus 18; Melanoma; Physical examination
\end{abstract}

\section{INTRODUCTION}

Cutaneous Bowen's disease (BD) is a squamous cell carcinoma in situ with only $2 \%$ of pigmented cases reported. ${ }^{1}$ Not limited to the skin, it can affect the mucous membranes and nail apparatus. Its occurrence on the fingers is even more exceptional. ${ }^{2,3}$

Proposed etiologies for this carcinoma in situ include sun damage and human papillomavirus (HPV) infection..$^{1-5}$ BD caused by sun damage is more frequent in light-skinned and older individuals, and may exhibit single or multiple lesions that are most frequently located on photoexposed areas. On the other hand, BD due to HPV infection is more frequent in the anogenital region of young or immunosuppressed adults, which is clinically characterized by papules or darkened plaques with irregular surface, usually caused by high-risk strains of HPV, such as $16.4,5$

BD dermoscopy was first described in 2004 by Argenziano et al., ${ }^{6}$ who observed that the presence of glomerular vessels was a suggestive finding of the disease. More recently, the presence of hypopigmented areas with disorganized architecture, linearly arranged brown or gray dots, and glomerular vessels, also arranged in a linear fashion, was described as a suggestive finding of pigmented Bowen's Disease. ${ }^{7-9}$
We report a case in which pigmented Bowen's disease simulated an extensive superficial melanoma on the left third finger. This manifestation and its dermoscopic findings show the importance of considering it as a differential diagnosis of melanocytic lesions, including melanoma.

\section{CASE REPORT}

We report a 57-year-old Asian male patient with no comorbidities and no previous history or complaints of genital lesions. Physical examination revealed a single irregular macula on the middle third of the left third finger, measuring $0.6 \mathrm{~cm}$ in diameter. The lesion had irregular limits with heterogeneous color, ranging from light brown to blackish-brown. It was asymptomatic featuring a slow growth rate in the past few months (Figure 1).

Dermoscopic examination showed a pigmented lesion of asymmetric disposition composed of an irregular network with rough radial streaming and ovoid structures in the periphery considered as globules. Its heterogeneous coloration varied from light brown to dark brown with whitish regions, mimicking regression areas. We observed no glomeruloid vessels or keratotic crusts (Figure 2).

Received on 31.07.2016.

Approved by the Advisory Board and accepted for publication on 23.11.2016

Work performed at Hospital da Santa Casa de Misericórdia de São Paulo - São Paulo (SP), Brazil.

Financial support: none.

Conflict of interest: none.

Pathology Laboratory at Santa Casa de Misericórdia de São Paulo - São Paulo (SP), Brazil.

Dermatology clinic at Santa Casa de Misericórdia de São Paulo - São Paulo (SP), Brazil

Private clinic - Piracicaba (SP), Brazil.

(C)2017 by Anais Brasileiros de Dermatologia 

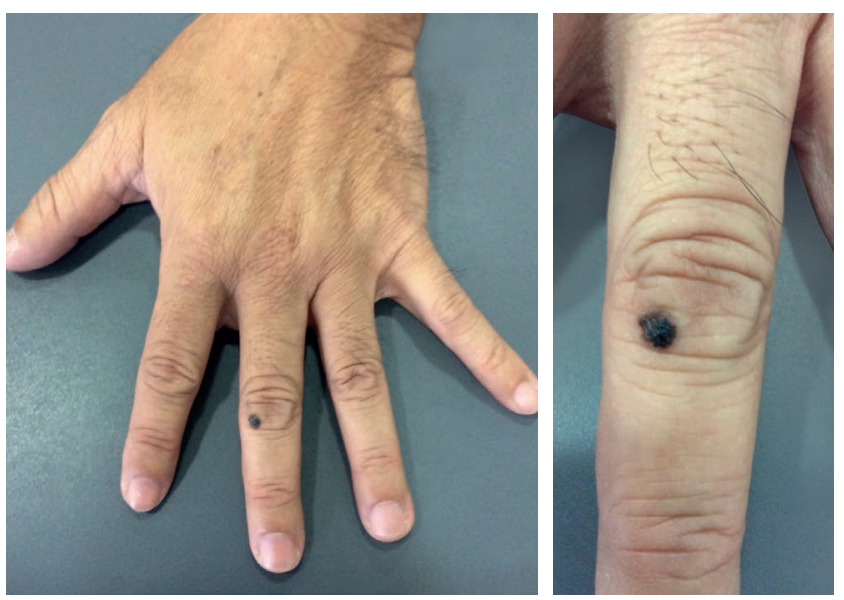

Figure 1: Dermatosis located on the dorsal aspect of the left $3^{\text {rd }}$ finger near the proximal interphalangeal joint characterized by a blackish macula with irregular borders

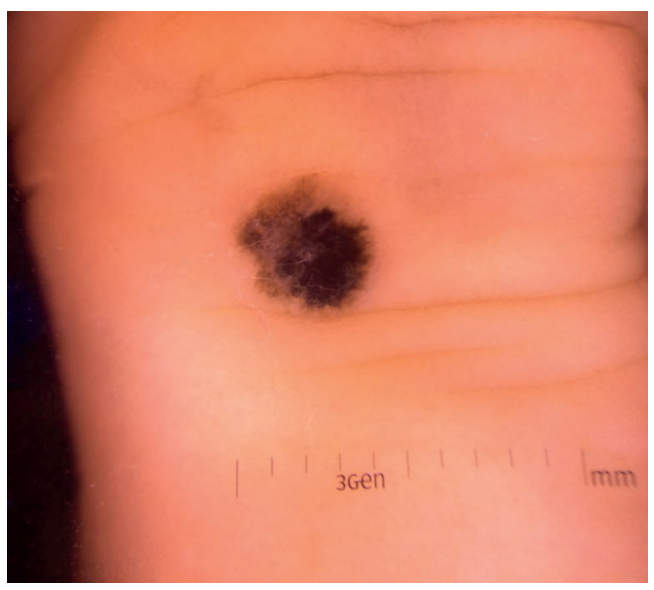

FiguRE 2: Dermoscopy reveals the asymmetry of the lesion with heterogeneous staining exhibiting heterogeneous coloration varying from light brown to dark brown with whitish regions. Note the peripheral structures that resemble an irregular network with loss of structures and rare streaming

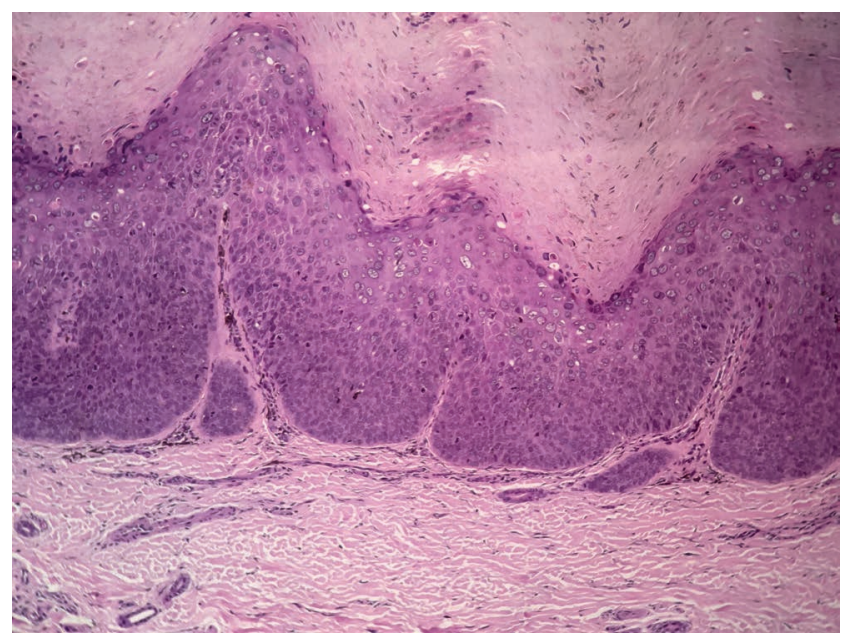

FIGURE 3: The histological section stained with Hematoxylin \& eosin (X100) shows papillomatosis and hyperparakeratosis with deposit of melanin in the stratum corneum. The epidermis is totally composed of atypical keratinocytes, some pigmented. The dermis also presents melanophages, justifying the color variation in dermoscopy
Clinical and dermoscopic features suggested the clinical hypothesis of superficial extensive melanoma. Excisional biopsy of a histological section stained with HE showed papillomatosis and hyperparaceratosis with deposition of melanin in the stratum corneum. The epidermis was totally composed of atypical keratinocytes, some pigmented. The dermis also revealed melanophages (Figures 3 and 4). The lesion was fully excised with no neoplastic compromising of surgical margins.

Based on this examination, we reached the diagnosis of pigmented squamous cell carcinoma in situ or pigmented Bowen's disease. The diagnosis was complemented by in situ hybridization, which confirmed the presence of high-risk HPV types $(16 / 18 / 31 / 33 / 35 / 39)$.

\section{DISCUSSION}

Bowen's disease is related to chronic exposure to various risk factors such as ultraviolet radiation, trauma, arsenic, immunosuppression, and viral infections. ${ }^{1,2}$

$\mathrm{HPV}$ infection is relevant in carcinomas of the anogenital and periungual regions. ${ }^{5}$ There are also reports that associate HPV with tumors of the fingers and others demonstrating that patients with genital lesions may develop periungual neoplasias. ${ }^{5}$ In the present case, the patient had neither a history nor presence of anogenital lesions on physical examination, which leads to the hypothesis that he became infected after contact with a positive high-risk HPV person.

It is characterized by slow-growing erythematous non-pigmented scaly plaques with well-defined borders. ${ }^{2}$ Histologically, it shows marked anaplasia of neoplastic keratinocytes associated with dyskeratosis, hyperkeratosis, parakeratosis, and architectural derangement of the epidermis that may or may not contain melanin. ${ }^{7,8}$ About $5 \%$ of these lesions exhibit dermal invasion if they are not properly treated, and $30 \%$ have metastatic potential at the time of excision. ${ }^{10}$

Pigmented Bowen's disease present itself as heterogeneous brown plaques, which may be keratotic or verrucous. The most common differential diagnoses include seborrheic keratosis,

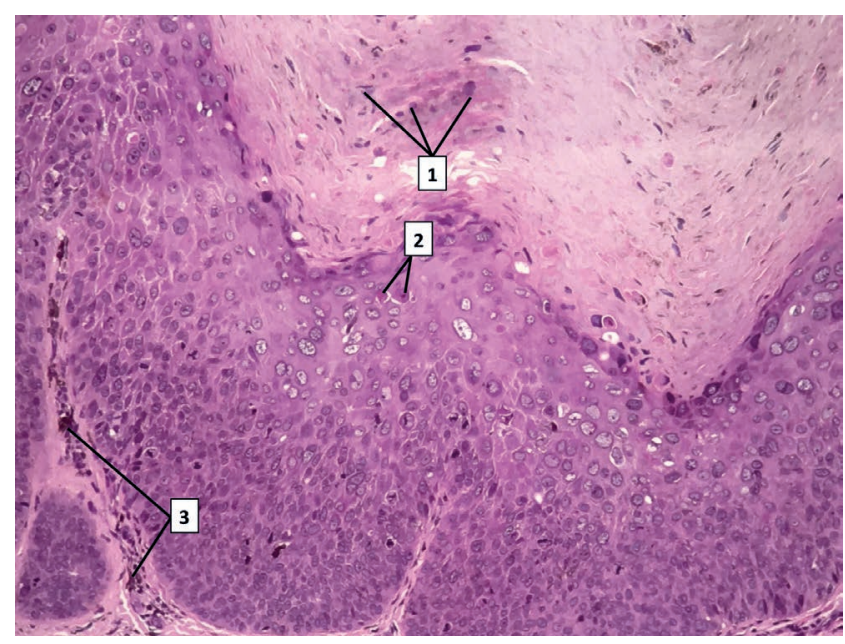

Figure 4: Histological section stained with Hematoxylin \& eosin (X200) detailing the corneal layer with melanin (1), apoptotic keratinocytes (2), and melanophages in the dermis (3) 
solar lentigo, pigmented actinic keratosis, melanocytic nevus, and melanoma. ${ }^{9}$ The pigmentation of these neoplasias is not related to prognosis and is probably due to a direct reflection of the presence of melanin in the keratinocytes that initiated the proliferation, as it happens in solar melanoses and seborrheic keratosis. ${ }^{1,2}$

Dermoscopic evaluation in Bowen's disease may be useful considering the absence of network and globules, in addition to the presence of keratinized crusts and glomerular vessels. However, pigmented cases may exhibit structures similar to small brown globules and/or loss of structures with homogeneous pigmentation. ${ }^{8,9}$

Our case report shows mistaken dermoscopic findings that are not clear enough to lead to a correct diagnostic conclusion, as already described in the literature. Although dermoscopy should always be performed with a view to improving preoperative diagnostic acuity, it may not be conclusive in some cases, corroborating that histology proves to be the "gold standard" for final diagnosis.

\section{REFERENCES}

1. Firooz A, Farsi N, Rashighi-Firoozabadi M, Gorouhi F. Pigmented Bowen's Disease of the Finger Mimicking Malignant Melanoma. Arch Iran Med. 2007;10:255-7.

2. Stante M, de Giorgi V, Massi D, Chiarugi A, Carli P. Pigmented Bowen's disease mimicking cutaneous melanoma: clinical and dermoscopic aspects. Dermatol Surg. 2004;30:541-4

3. Ramirez-Fort MK. Case report: human papillomavirus induced periungual pigmented Bowen's disease. Dermatol Pract Concept. 2012;2:57-9.

4. Murao K, Tetsutani M, Ishigami T, Kubo Y, Arase S. Bowen disease of the palm associated with human papillomavirus 52. Clin Exp Dermatol. 2013;38:489-91.

5. Shim WH, Park HJ, Kim HS, Kim SH, Jung DS, Ko HC, et al. Bowenoid papulosis of the vulva and subsequent periungual Bowen's disease induced by the same mucosal HPVs. Ann Dermatol. 2011;23:493-6.

6. Argenziano G, Zalaudek I, Corona R, Sera F, Cicale L, Petrillo G, et al. Vascular structures in skin tumors. A dermoscopy study. Arch Dermatol. 2004;140:1485-9.

7. Hu SC, Chiu HH, Chen GS, Ke CL, Cheng ST. Dermoscopy as a diagnostic and follow-up tool for pigmented Bowen's disease on acral region. Dermatol Surg. 2008;34:1248-53.

8. Cameron A, Rosendahl C, Tschandl P, Riedl E, Kittler H. Dermatoscopy of pigmented Bowen's disease. J Am Acad Dermatol. 2010;62:597-604.

9. Ishioka P, Yamada S, Michalany NS, Hirata SH. Dermoscopy of Bowen's disease: pigmented variant on the penis. An Bras Dermatol. 2012;87:482-4.

10. Wilmer EM, Lee KC, Higgins W 2nd, Cruz AP. Hyperpigmented palmar plaque: an unexpected diagnosis of Bowen disease. Dermatol Online J. 2013;19:18573.
MAILING ADDRESS:

John Verrinder Veasey

Rua Dr. Cesário Mota Jr., 112

Vila Buarque

01221-020 São Paulo, SP - Brazil

E-mail:johnveasey@uol.com.br

How to cite this article: Lellis RF, Veasey JV, Gonçalves RDJ. Pigmented Bowen's disease associated with high-risk HPV simulating melanoma of the hand. An Bras Dermatol. 2017;92(5):686-8. 